\title{
Qualitative assessments of myocardial ischemia by cardiac MRI and coronary stenosis by invasive coronary angiography in relation to quantitative perfusion by positron emission tomography in patients with known or suspected stable coronary artery disease
}

\author{
Shahnaz Akil, PhD, ${ }^{\mathrm{a}, \mathrm{b}}$ Fredrik Hedeer, MD, ${ }^{\mathrm{a}}$ Marcus Carlsson, MD, PhD, ${ }^{\mathrm{a}}$ \\ Håkan Arheden, $\mathrm{MD}, \mathrm{PhD},{ }^{\mathrm{a}}$ Jenny Oddstig, $\mathrm{PhD},{ }^{\mathrm{c}}$ Cecilia Hindorf, $\mathrm{PhD},{ }^{\mathrm{c}}$ \\ Jonas Jögi, $\mathrm{MD}$, PhD, ${ }^{\mathrm{a}}$ David Erlinge, $\mathrm{MD}, \mathrm{PhD},{ }^{\mathrm{d}}$ and Henrik Engblom, $\mathrm{MD}, \mathrm{PhD}^{\mathrm{a}}$ \\ a Department of Clinical Sciences Lund, Clinical Physiology, Skane University Hospital, Lund \\ University, Lund, Sweden \\ b Department of Radiological Sciences, College of Health and Rehabilitation Sciences, Princess \\ Nourah bint Abdulrahman University, Riyadh, Saudi Arabia \\ c Radiation Physics, Skåne University Hospital, Lund, Sweden \\ d Department of Clinical Sciences Lund, Department of Cardiology, Skane University Hospital, \\ Lund University, Lund, Sweden
}

Received Jun 26, 2018; accepted Nov 26, 2018

doi: $10.1007 / \mathrm{s} 12350-018-01555-1$

Background. To relate findings of qualitative evaluation of first-pass perfusion-CMR and anatomical evaluation on coronary angiography $(\mathrm{CA})$ to the reference standard of quantitative perfusion, cardiac PET, in patients with suspected or known stable coronary artery disease (CAD).

Methods and Results. Forty-one patients referred for CA due to suspected stable CAD, prospectively performed adenosine stress/rest first-pass perfusion-CMR as well as ${ }^{13} \mathrm{~N}-\mathrm{NH}_{3}$ PET on the same day, $4 \pm 3$ weeks before CA. Angiographers were blinded to PET and CMR results. Regional myocardial flow reserve (MFR) $<2.0$ on PET was considered pathological. Vessel territories with stress-induced ischemia by CMR or vessels with stenosis needing revascularization had a significantly lower MFR compared to those with no regional stressinduced ischemia or vessels not needing revascularization $(P<0.001)$. In 4 of 123 vessel territories with stress-induced ischemia by CMR, PET showed a normal MFR. In addition, 12 of 123 vessels that underwent intervention showed normal MFR assessed by PET.

Conclusion. The limited performance of qualitative assessment of presence of stable CAD with CMR and $\mathrm{CA}$, when related to quantitative ${ }^{13} \mathrm{~N}-\mathrm{NH}_{3}$ cardiac PET, shows the need for fully quantitative assessment of myocardial perfusion and the use of invasive flow reserve measurements for $\mathrm{CA}$, to confirm the need of elective revascularization. (J Nucl Cardiol 2020;27:2351-9.)

Key Words: Cardiac magnetic resonance imaging $•$ cardiac positron emission tomography • coronary angiography $\cdot$ coronary artery disease $\cdot$ myocardial flow reserve

Electronic supplementary material The online version of this article (https://doi.org/10.1007/s12350-018-01555-1) contains supplementary material, which is available to authorized users.

The authors of this article have provided a PowerPoint file, available for download at SpringerLink, which summarises the contents of the paper and is free for re-use at meetings and presentations. Search for the article DOI on SpringerLink.com.
Reprint requests: Shahnaz Akil, $\mathrm{PhD}$, Department of Clinical Sciences Lund, Clinical Physiology, Skane University Hospital, Lund University, 22185 Lund, Sweden; Shahnaz.akil@med.lu.se $1071-3581 / \$ 34.00$

Copyright $(\subset 2018$ The Author(s) 


\begin{tabular}{|ll|}
\hline Abbreviations & \\
AHA & American Heart Association \\
CAD & Coronary Artery Disease \\
CA & Coronary Angiography \\
CABG & Coronary artery bypass grafting \\
CMR & Cardiac magnetic resonance imaging \\
ESC & European Society of Cardiology \\
FFR & Fractional flow reserve \\
MFR & Myocardial flow reserve \\
MP & Myocardial Perfusion \\
PET & Positron Emission Tomography \\
PCI & Percutaneous coronary intervention \\
\hline
\end{tabular}

See related editorial, pp. 2360-2364

\section{INTRODUCTION}

Coronary artery disease (CAD) is the leading cause of mortality worldwide. Therefore, accurate methods for diagnosing $\mathrm{CAD}$ are desirable. In the case of stable CAD, myocardial perfusion (MP) is affected predominantly during stress due to one or several flowlimiting stenoses, in the coronary arteries, resulting in stress-induced ischemia.

In patients with suspected stable $\mathrm{CAD}$, assessment of presence and severity of stress-induced ischemia is of importance for prognosis. ${ }^{1-3}$ In addition, current guidelines from the European Society of Cardiology (ESC) and the American Heart Association (AHA) recommend the evaluation of stress-induced ischemia non-invasively, in patients with intermediate risk of stable CAD, before taking decisions regarding revascularization. ${ }^{1,4,5}$ Fractional flow reserve (FFR) assessment of intermediate stenoses found on coronary angiography (CA) is also recommended before an intervention since coronary angiography alone has been shown to have low diagnostic accuracy compared to FFR. ${ }^{6}$ Despite this knowledge and the current recommendations, coronary interventions are still performed in many patients based on the presence of anatomically significant stenosis on the $\mathrm{CA}$, clinical status and risk factors ${ }^{7}$ without any prior assessment of stress-induced ischemia. ${ }^{8,9}$

First-pass cardiac magnetic resonance imaging (CMR) is one of the non-invasive methods available for the assessment of stress-induced ischemia. In clinical routine and in large clinical studies, qualitative visual assessment of the regional MP distribution is usually performed. ${ }^{10-13}$ Semi-quantitative methods with CMR are not as widely used, while the fully quantitative approaches are still being developed and validated. ${ }^{14-16}$

Dynamic cardiac positron emission tomography (PET) is another non-invasive method and considered the reference method for assessment of stress-induced ischemia because of its ability to quantify regional and global MP ( $\mathrm{ml} / \mathrm{min} / \mathrm{g})$ at rest and stress and thereby myocardial flow reserve (MFR).

Therefore, the aim of this study was to relate findings of qualitative evaluation of first-pass perfusionCMR and clinical assessment of coronary angiography (CA) to quantitative perfusion assessed by cardiac PET in patients with suspected or known stable coronary artery disease $(\mathrm{CAD})$.

\section{METHODS}

\section{Study Population}

A total of 41 patients ( 9 females, mean age 67 years \pm 7 , age range 50-86 years) with suspected or known stable CAD, clinically referred for $\mathrm{CA}$ in conjunction with possible elective percutaneous coronary intervention (PCI), were prospectively included from November, 2013 to December, 2016. Patients were referred for $\mathrm{CA}$ in conjunction with possible PCI due to experience of stable angina symptoms in addition to clinical history wih risk factors in the majority of patients $(80 \%)$ and due to results of prior non-invasive stress-testing in the minority of patients $(20 \%)$. Patients with atrial fibrillation, known chronically occluded vessels, claustrophobia, asthma, severe chronic obstructive lung disease, and glomerular filtration rate $<30 \mathrm{ml} / \mathrm{min}$ were excluded. The patients performed stress/rest first-pass perfusion-CMR (1.5 T) as well as ${ }^{13} \mathrm{~N}-\mathrm{NH}_{3}$ cardiac PET on the same day (4-5 hour apart), with the same sequence in all patients, at mean $4 \pm 3$ weeks (0.5-14 weeks) before CA. The CMR and cardiac PET examinations were performed for the purpose of the present study and kept blinded to the referring physician and the angiographer. The study was approved by the Regional Ethical Committee, Lund, Sweden and a written informed consent was obtained from all patients.

\section{Cardiac PET Imaging and Reconstruction}

All patients performed the rest imaging first, followed by stress imaging approximately 1 hour later. At rest, patients were injected with ${ }^{13} \mathrm{~N}-\mathrm{NH}_{3}$ at a constant flow rate. For the stress examination, ${ }^{13} \mathrm{~N}-\mathrm{NH}_{3}$ was injected, at the same flow rate, 3 minutes after the beginning of adenosine infusion $(140 \mu \mathrm{g} / \mathrm{kg} / \mathrm{min})$. Adenosine infusion continued, with continuous ECG monitoring, for another 4 minutes after isotope injection since the data from the first 4 minutes after isotope injection are used for calculation of the quantitative myocardial perfusion (see below). The injected activity of ${ }^{13} \mathrm{~N}^{-\mathrm{NH}_{3}}$ was $525 \pm 78 \mathrm{MBq}$ at both rest and stress.

For positioning, a scout view over the chest was performed followed by a low-dose computed tomography for attenuation correction $(120 \mathrm{kV} ; 10 \mathrm{mAs}, 10$; rotation time 0.5 second). The PET acquisition was started simultaneously with the isotope injection, for both rest and stress examinations. Images were acquired using a GE Discovery 690 PET/ CT with an acquisition time of 15 minutes for both stress and rest PET image acquisition. 
Before reconstructing the images, evidence for patient motion was checked between the CT and PET images and manual adjustments were made. To ensure correction for the increase in accumulation of the metabolic product $\left({ }^{13} \mathrm{~N}\right.$ glutamine) trapped in the myocardial tissue, only the first 4 min of the PET acquisition were used, as recommended by DeGrado et al. ${ }^{17}$ to reconstruct the rest and stress dynamic images into 15 time-frames $(12 \times 10 \mathrm{~s}, 2 \times 30 \mathrm{~s}$ and $1 \times 60 \mathrm{~s})$ using OSEM (3 iterations, 12 subsets) and a $5 \mathrm{~mm}$ post-filter.

\section{Cardiac PET Image Analysis}

The reconstructed dynamic images were analyzed using the software Carimas (version 2.7, Turku, Finland). The left ventricle was delineated automatically with manual adjustments when needed. The activity in the blood and the myocardial wall as a function of time served as input information to the DeGrado compartment $\operatorname{model}^{17}$ for ${ }^{13} \mathrm{~N}$ $\mathrm{NH}_{3}$, allowing quantification of the rest and stress global MP in $\mathrm{ml} / \mathrm{min} / \mathrm{g}$ as well as the regional MP in each of the three vessel territories (LAD, LCX, RCA). The regional MFR was calculated by dividing the stress by the rest regional MP. MFR $<2.0$ was considered pathologic as previously suggested. ${ }^{18,19}$

\section{CMR Imaging and Reconstruction}

Patients underwent perfusion-CMR in either a $1.5 \mathrm{~T}$ Philips Achieva (Best, The Netherlands; $n=11$ ) or Siemens, Magnetom Aera (Erlangen, Germany; $n=30$ ) as the CMR scanner was replaced at the hospital during the study.

Three short-axis slices (basal, mid-ventricular, and apical) were acquired at rest and after 3 minutes of adenosine infusion $(140 \mu \mathrm{g} / \mathrm{kg} / \mathrm{min})$, during the first-pass of $0.05 \mathrm{mmol} / \mathrm{kg}$ bolus of the gadolinium-based contrast agent Dotarem (Guerbet, Roissy, France). For the Philips scanner spatial resolution was $2 \times 2 \times 10 \mathrm{~mm}$ reconstructed to $1.4 \times 1.4 \times 10 \mathrm{~mm}$ and accelerating factor 3 . For the Siemens scanner spatial resolution was $1.9 \times 2.4 \times 8 \mathrm{~mm}$ reconstructed to $1.9 \times 1.9 \times 8 \mathrm{~mm}$ and accelerating factor 3 . Late gadolinium enhancement imaging (LGE) was performed to evaluate presence of infarction and fibrosis.

\section{CMR Image Analysis}

All CMR analysis was performed using the software Segment version 2.0 (http://segment.heiberg.se) ${ }^{20}$ All stress/ rest first-pass perfusion images were visually assessed for presence of stress-induced ischemia, by an experienced physician (HE) who was blinded to cardiac PET and CA results. A second experienced observer (MC), who was blinded to the results by the first experienced observer (HE) as well as to cardiac PET and CA results, re-assessed the first-pass CMR images of 14/41 patients which were randomly selected from the included population. A hypo-enhanced area at stress, present during at least three heart cycles, but not present at rest and not corresponding to contrast enhancement on LGE, indicated stress-induced ischemia. Stress-induced ischemia was assigned to one of the three vessel territories (LAD, LCX, and RCA). LGE images were qualitatively assessed for presence of infarct or fibrosis. Scar size was determined from the LGE short-axis images using a recently described automated algorithm for scar quantification taking signal intensity distribution, coronary vessel territory, and partial volume effects into consideration. ${ }^{21}$

\section{Coronary Angiography}

Angiographers were blinded to cardiac PET and CMR, which were strictly research-initiated examinations not asked for by the clinician. This design of the study allowed for assessment of how well clinical routine, often to a large extent dependent on assessing angiograms, can guide accurate treatment decisions. The angiographers performed the CA according to clinical routine (fractional flow reserve measurements performed only at the angiographer's request). Angiograms were visually assessed by the cardiologist responsible for writing the clinical angiography report, not aware of any of the PET or CMR results. The angiograms were assessed according to the clinical routine taking available clinical data and patient history into account. Thus, no specific percentage of stenosis was considered significant, just the judgment of the angiographer performing the angiography, with or without iFR/FFR. Instantaneous wave-free ratio (iFR) $>0.89$ and FFR $>0.80$ were considered normal, as previously suggested. ${ }^{22,23}$ Flow reserve measurements (iFR or FFR) were performed in $17 / 123$ vessels (14 iFR and 3 FFR) belonging to $15 / 41$ patients.

\section{Statistical Analysis}

Results are given in mean $\pm \mathrm{SD}$. A non-paired $t$ test was used for assessment of differences in regional MFR by PET between normal vessels and vessels requiring revascularization by $\mathrm{CA}$ as well as between normal vessel territories and territories with stress-induced ischemia by first-pass CMR. All graphs were generated with the software Graphpad Prism version 6.07 (Graph Pad Software, Inc., La Jolla, CA, USA). Results with $P$ values $<0.05$ were considered statistically significant.

\section{RESULTS}

Characteristics of the included patients can be found in Table 1 and characteristics of the electively revascularized patients $(n=24)$ are shown in Table 2. Twentyfour of the 41 patients were revascularized, 20 by PCI and 4 by coronary artery bypass graft surgery. The CABG patients were not analyzed separate from the PCI patients. History of previous PCI was found in 11/24 revascularized patients and in 4/17 non-revascularized patients. The majority of the electively revascularized patients had single-vessel disease (19/24). Infarct by LGE was found in 15/41 patients (6 LAD, 8 RCA, 6 LCX) with a scar size of $9 \pm 6 \%$. Septal fibrosis of nonischemic origin was detected in $1 / 41$ patients. In the 
Table 1. Baseline characteristics of the included patients $(n=41)$

\begin{tabular}{lll}
\hline Age & $67 \pm 7$ & \\
Females & 9 & $22 \%$ \\
BMI $\left(\mathrm{kg} / \mathrm{m}^{2}\right)$ & $27 \pm 3$ & \\
Smoker & 5 & $12 \%$ \\
Previous smoker & 21 & $51 \%$ \\
Prior PCI & 17 & $41 \%$ \\
Prior CABG & 3 & $7 \%$ \\
Prior myocardial infarction & 11 & $27 \%$ \\
Diabetes & 9 & $22 \%$ \\
Hypertension & 29 & $71 \%$ \\
Hypercholesterolemia & 27 & $66 \%$ \\
Heredity for coronary artery disease & 9 & $22 \%$ \\
Betablockers & 23 & $56 \%$ \\
ACE-inhibitor/ARB & 19 & $46 \%$ \\
Statins & 36 & $88 \%$ \\
Anti-coagulants & 35 & $85 \%$ \\
\hline
\end{tabular}

$B M I$, body mass index; $P C I$, percutaneous coronary intervention; $C A B G$, coronary artery bypass graft; $A C E$, angiotensin converting enzyme, $A R B$, angiotensin II receptor blockers; $L G E$, late gadolinium enhancement

Table 2. Characteristics by coronary angiography of the electively revascularized patients $(n=24)$

\begin{tabular}{lll}
\hline 1 -vessel & 19 & $79 \%$ \\
2-vessels & 3 & $13 \%$ \\
3 -vessels & 2 & $8 \%$ \\
LAD & 15 & $63 \%$ \\
LCX & 7 & $29 \%$ \\
RCA & 9 & $38 \%$ \\
\hline
\end{tabular}

subset of first-pass CMR images which were assessed by two experienced observers (14/41), the assessments corresponded to each other in $100 \%$ of the cases regarding presence or absence of stress-induced ischemia. The hemodynamic response (blood-pressure and heart rate response) during stress CMR and cardiac PET is presented in Table 3. MP at rest did not exceed $1.4 \mathrm{ml} / \mathrm{min} / \mathrm{g}$ for any patient.

\section{CMR in Relation to Cardiac PET}

There was significantly lower MFR in vessel territories with stress-induced myocardial ischemia by CMR $(1.6 \pm 0.5)$ compared to those with no regional stress-induced ischemia $(2.4 \pm 0.8)(P<0.001$, Figure 1A). CMR had a sensitivity of $47 \%$ and a specificity of $75 \%$ on a per-patient basis, and a sensitivity of $27 \%$ and a specificity of $96 \%$ on a per-vessel basis for MFR $<2.0$ by PET (Table 4 ). Thus, there were several vessel territories $(37 / 123,30 \%)$ with decreased MFR $(<2.0)$ but normal first-pass perfusion by CMR. Furthermore, three vessel territories with stress-induced ischemia by first-pass perfusion-CMR had MFR $>2.0$ $(2.3 \pm 0.2$, range $2.1-2.5$, Figure $1 \mathrm{~A})$. The experienced CMR observer reported low image quality in the images belonging to two of these vessel territories.

\section{CA in Relation to Cardiac PET}

There was significantly lower MFR in vessel territories supplied by revascularized arteries (1.9 \pm $0.6)$ compared to non-revascularized arteries $(2.4 \pm 0.8)$ $(P<0.001$, Figure 1B). CA had a sensitivity of $76 \%$ and a specificity of $54 \%$ on a per-patient basis, and a sensitivity of $39 \%$ and a specificity of $83 \%$ on a pervessel basis, for MFR $<2.0$ by PET (Table 4 ). Thus, there were several vessel territories with decreased MFR $(<2.0)$ but arteries evaluated by the angiographer as not

Table 3. Hemodynamic response during CMR and PET

\begin{tabular}{lcccccccc}
\hline & \multicolumn{3}{c}{ At rest } & & \multicolumn{3}{c}{ Adenosine } \\
\cline { 2 - 3 } & CMR & Cardiac PET & P-value & & CMR & Cardiac PET & P-value \\
\hline Heart rate (beats per minute) & $68 \pm 9$ & $66 \pm 7$ & 0.5 & & $82 \pm 11$ & $83 \pm 10$ & 0.8 \\
Systolic pressure (mmHg) & $138 \pm 17$ & $149 \pm 17$ & 0.004 & & $134 \pm 27$ & $135 \pm 19$ & 0.7 \\
Diastolic pressure (mmHg) & $80 \pm 12$ & $80 \pm 16$ & 0.7 & & $76 \pm 16$ & 0 0* * & NA \\
\hline
\end{tabular}

${ }^{*}$ Mean of the average heart rate during adenosine

for each patient

* * Not measured during adenosine cardiac PET due to use of doppler instead of blood-pressure cuff 

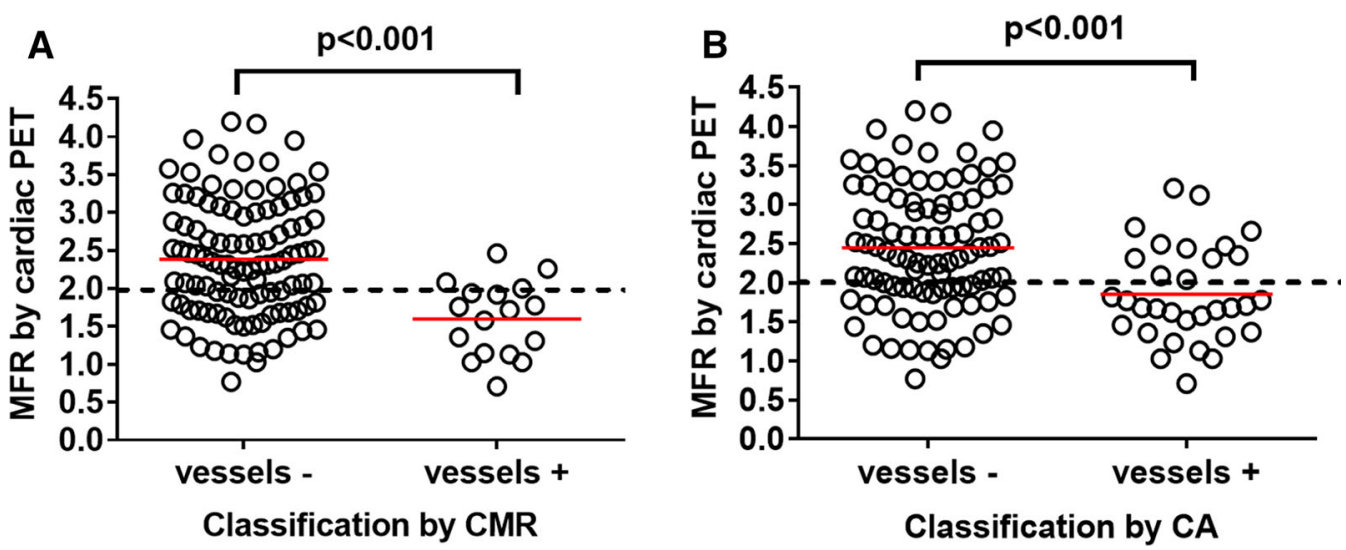

Figure 1. Myocardial flow reserve (MFR) by cardiac positron emission tomography (PET) for a total of 123 coronary arteries (41 patients X 3) classified by (A) cardiac magnetic resonance imaging (CMR) as normal (vessels -) or supplying territories with stress-induced myocardial ischemia (vessels + ) $(\mathbf{B})$ coronary angiography $(\mathrm{CA})$ as normal/not needing revascularization (vessels -) or with stenosis that was electively revascularized (vessels + ). Red lines indicate mean MFR and the dashed line indicates MFR cut-off at 2.0.

Table 4. Diagnostic accuracy of $C A$ versus $C M R$, on a per-patient and per-vessel or -vessel territory analysis, using MFR by cardiac PET as reference

\begin{tabular}{lccccc}
\hline & \multicolumn{2}{c}{ Per-patient } & & \multicolumn{2}{c}{ Per-vessel or -vessel territory } \\
\cline { 2 - 3 } \cline { 5 - 6 } & CA (\%) & CMR (\%) & & CA (\%) & CMR (\%) \\
\hline Sensitivity & 76 & 47 & & 39 & 27 \\
Specificity & 54 & 75 & & 83 & 96 \\
PPV & 54 & 57 & & 63 & 82 \\
NPV & 76 & 67 & 66 & 65 \\
\hline
\end{tabular}

needing revascularization $(31 / 123,25 \%)$. Furthermore, several vessels $(12 / 123,10 \%)$ that underwent intervention, after being evaluated by the angiographer as needing revascularization, had MFR $>2.0(2.5 \pm 0.4$, range 2.04-3.0, Figure 1B). No invasive fractional flow reserve measurements were performed on these vessels.

\section{Invasive CA Measurements in Relation to Cardiac PET and CMR}

In 16 of the 17 vessel territories where iFR or FFR were used, the iFR/FFR findings corresponded to the MFR findings by PET. A normal iFR/FFR was found in $12 / 12$ vessel territories with MFR $>2.0$. A decreased $\mathrm{iFR} / \mathrm{FFR}$ was found in $4 / 5$ vessel territories with MFR $<$ 2.0. In one vessel territory with MFR $<2.0(\mathrm{MFR}=1.5)$, $\mathrm{iFR}$ was normal $(\mathrm{iFR}=1.0)$.
In 12 of the 17 vessel territories where iFR or FFR were used, the iFR/FFR findings corresponded to qualitative assessment by CMR. A normal iFR/FFR was found in 11/16 vessel territories with normal CMR. A decreased iFR/FFR was found in 1/1 vessel territory with pathologic CMR.

\section{Qualitative CMR and CA Versus Quantitative Cardiac PET}

Two of the three vessel territories with stressinduced ischemia by CMR but MFR $>2.0(2.3 \pm 0.19$, range 2.1-2.5) were supplied by vessels which underwent revascularization due to CA findings.

Figure 2 shows an example of a patient where findings from CMR, CA and quantitative cardiac PET did not correspond to each other. The cardiac PET rest/ 
A

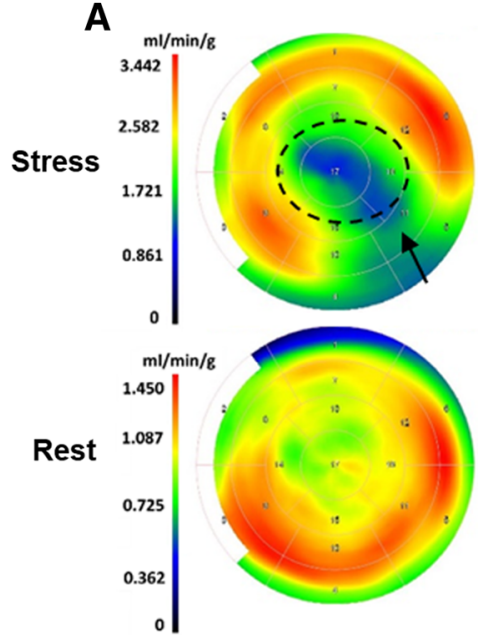

B
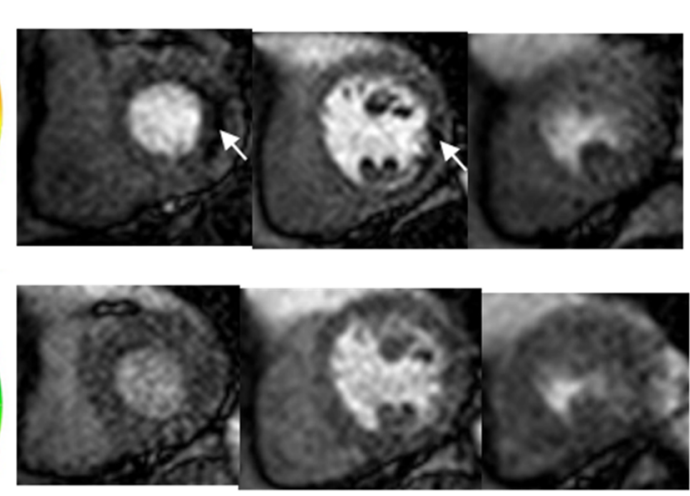

C

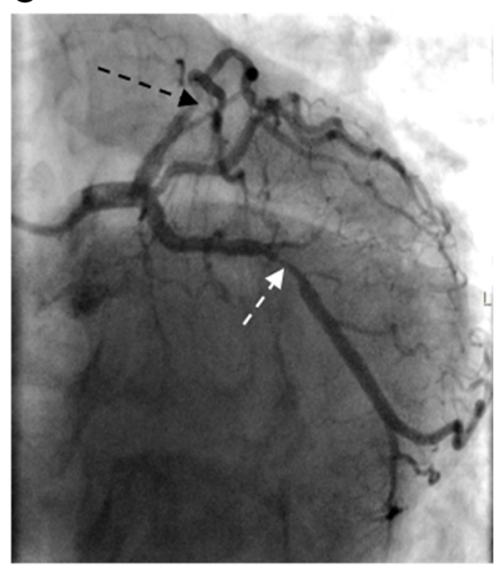

Figure 2. An example of a patient with a cardiac positron emission tomography (A) revealing abnormal myocardial blood flow at stress in both the LCX (black arrow) and LAD vessel territories (dashed circle). The bull's eye plots represent the distribution of the quantified absolute myocardial blood flows $(\mathrm{ml} / \mathrm{min} / \mathrm{g}$ tissue) in the left ventricle. The color scales to the left of the bull's eyes represent the flow ranges with warm colors indicating higher flows and cold colors lower flows. In this case first-pass perfusion-CMR (B) showed stress-induced ischemia only in the LCX territory (white arrows) while the angiogram $(\mathbf{C})$ revealed a stenosis, evaluated as needing revascularization, in the LAD (dashed black arrow), not seen by CMR, in addition to a stenosis evaluated as needing revascularization in LCX (dashed white arrow).

stress bull's eye plots show that the patient has 2-vessel disease (LCX, LAD), while the qualitative assessment of the first-pass CMR images reveal stress-induced ischemia in one vessel territory (LCX). The qualitative assessment of the CA revealed a stenosis evaluated as needing revascularization in the vessels supplying these territories with abnormal myocardial blood flow at stress by PET.

\section{DISCUSSION}

The present prospective study shows that the diagnostic accuracy of qualitative perfusion assessment with first-pass CMR or assessment with CA is limited in patients with suspected stable CAD, when considering quantitative cardiac ${ }^{13} \mathrm{~N}_{-} \mathrm{NH}_{3}$ PET as the reference standard. Given the significant number of patients and vessel territories with discrepancies between CMR or $\mathrm{CA}$ and MFR, there is a need for improvement in selection of patients with suspected CAD that should undergo elective revascularization. Furthermore, given that MFR also assesses microvascular disease, requiring a different treatment than epicardial coronary disease, there is a need for further studies of the myocardial pathophysiology underlying a MFR $<2.0$ to improve prognosis and quality of life in this group of patients.

\section{CMR in Relation to Cardiac PET}

In this study, the diagnostic accuracy for CMR in evaluating presence or absence of stable CAD, on both the per-patient and per-vessel analysis, was limited (Table 4). Previous studies have reported higher diagnostic accuracies for CMR, especially sensitivity and NPV, for the per-patient analysis ${ }^{10,12,13,24}$ when comparing to angiography. However, the majority of previously recorded diagnostic accuracies are based on a larger number of patients, different evaluation criteria for CMR and CA and a different study design, including $\geq 50 \%$ diameter stenosis on $\mathrm{CA}$ being the reference standard, as in the retrospective study by Jaarsma et al. Furthermore, for one previous study, ${ }^{10}$ the diagnostic accuracy of CMR in detecting angiographically defined CAD was based on presence of stress-induced ischemia on first-pass perfusion or presence of infarction/fibrosis on LGE or both.

Several vessel territories with decreased MFR, in the present study, had normal first-pass perfusion (37/ $123,30 \%$, Figure 1A). A possible explanation might be presence of left ventricular dysfunction, multi-vessel disease, or vascular disease not related to coronary stenosis, i.e. microvascular disease, and not affecting the relative in-flow kinetics in first-pass perfusion imaging. However, qualitative assessment by CMR has still been shown to be superior to other non-invasive imaging 
modalities (i.e. myocardial perfusion single photon emission tomography). ${ }^{6,10}$

In the current study, three of the vessel territories with normal MFR were classified as pathologic by CMR (Figures $1 \mathrm{~A}$ and 2). This might be explained by poor image quality on CMR (which was reported in one case) or dark rim artifacts shown to affect the diagnostic accuracy of first-pass perfusion-CMR. ${ }^{25}$

\section{CA in Relation to Cardiac PET}

In the present study, the diagnostic accuracy for CA in evaluating presence or absence of stable CAD, on both the per-patient and per-vessel analysis, was limited (Table 4). This is in line with the previously reported limited diagnostic accuracy of qualitative $\mathrm{CA},{ }^{6}$ but with a lower specificity for the per-patient analysis and a lower sensitivity for the per-vessel analysis found in the current study. However, in contrast to previous studies, ${ }^{6}$ a clinical decision of elective revascularization, and not a specific cut-off value of $\%$ diameter stenosis, was considered a positive finding in the current study.

Several vessels $(n=12)$ that underwent intervention, after being evaluated as having stenosis needing revascularization, had a normal MFR (Figure 1B), suggesting unnecessary revascularization of these vessels. In these vessels, no flow reserve measurements during CA were performed. The results in the current study, where evaluation of FFR or iFR was performed only in a minority of the patients $(15 / 41,37 \%)$, are thus in concordance with findings from a recent metaanalysis which concluded that qualitative CA had a lower diagnostic performance than other imaging modalities when related to invasive FFR measurements. ${ }^{6}$ The current study protocol included CA performed according to clinical routine, which did not require the angiographers to perform flow reserve measurements. For vessels where the angiographer decided to perform flow measurements during CA $(n=17)$, findings of MFR by cardiac PET corresponded to those of iFR/FFR in 16/ 17 vessel territories. Thus, the findings in the present study further emphasize what has previously been shown about the importance of the functional significance of a stenosis, ${ }^{26}$ and further encourage the use of flow reserve measurements during $\mathrm{CA}$ in patients with suspected significant stenosis.

Moreover, only 2/12 vessels, which had normal MFR but underwent intervention, due to presence of stenosis evaluated as needing revascularization by CA, showed stress-induced ischemia by CMR in the territories they supplied. This further highlights the need of using non-invasive stress-testing in patients with suspected CAD, to decrease the number of unnecessary $\mathrm{CA}$ and revascularizations, as recommended by the ESC and AHA guidelines. ${ }^{4}$ Furthermore, in the current study, several vessel territories $(19 / 50,38 \%$, Figure 1B) with pathologic MFR had coronary stenosis not needing revascularization. Presence of microvascular disease can be a possible explanation for decreased MFR not detected by qualitative CA.

\section{MFR by Cardiac PET}

The cut-off value of $<2.0$ for ${ }^{13} \mathrm{~N}_{-}-\mathrm{NH}_{3}$ cardiac PET, for a pathologic MFR, has been previously used in several studies. ${ }^{18,19,27}$ A previous study by Stuijfzand et al. ${ }^{28}$ has suggested that the use of the absolute quantification of MP at stress (in $\mathrm{ml} / \mathrm{min} / \mathrm{g}$ ), instead of MFR, has similar diagnostic accuracy based on dynamic cardiac PET with $\left[{ }^{15} \mathrm{O}\right] \mathrm{H}_{2} \mathrm{O}$. However, there is a lack of studies investigating the diagnostic accuracy of cut-off values of absolute quantification of MP at stress (in $\mathrm{ml} /$ $\mathrm{min} / \mathrm{g}$ ) against a wider range of cut-off values of degree of stenosis by CA, including a population with suspected microvascular obstruction. Moreover, to the best of our knowledge, no cut-off value for absolute quantification of MP at stress using the radioisotope ${ }^{13} \mathrm{~N}_{-}-\mathrm{NH}_{3}$ is available in the published literature. Thus, cut-off values for quantification in $\mathrm{ml} / \mathrm{min} / \mathrm{g}$ using ${ }^{13} \mathrm{~N}-\mathrm{NH}_{3}$, as well as for other cardiac PET isotopes, are still debated and therefore we used MFR rather than absolute perfusion. MFR calculated from the regional stress and rest MP has been shown to be a sensitive measure to detect stable CAD and is valuable in the prediction of patient prognosis. ${ }^{18,19,29}$

Despite its quantitative ability, dynamic cardiac PET is not a widely used examination for the detection of stable CAD. The challenging logistics of the examination, which includes isotopes with short half-lives requiring the presence of an on-site cyclotron or isotope generator, make this examination costly. ${ }^{30}$

\section{Limitations}

The following limitations should be considered when interpreting the results in this study. (1) The binary MFR cut-off value of 2.0 has its limitations when used to assess physiological processes such as MP. However, this cut-off is clinically established and has been used in several previous studies. ${ }^{18,19,27}$ Furthermore, it is known 
that MFR could be decreased because of non- epicardial $\mathrm{CAD}$, such as microvascular disease, which $\mathrm{CA}$ and first-pass CMR cannot detect and which revascularization cannot treat. This limitation in using MFR as a reference could partly explain the low sensitivity found for both first-pass CMR and CA. Therefore, in the clinical routine quantitative and qualitative assessment of the myocardial perfusion should be combined for more accurate diagnosis. (2) The patients were included based on having a clinical referral for a CA with possible PCI, which means that the patients included in this study probably have a higher pre-test likelihood of CAD. Thus, these results can not be applied to patients with suspected CAD in general. (3) The use of CMR scanners from two different vendors might affect the qualitative assessment of first-pass CMR images due to difference in acquired spatial and temporal resolutions. (4) The majority of the revascularized patients had a stenosis evaluated as needing revascularization in the LAD (63\%) and most of the revascularized patients (79\%) had single-vessel disease by CA, which makes the generalization of the findings in the study somewhat limited for other coronary vessels and patients with multi-vessel disease. (5) The results might not be extrapolated to other PET isotopes such as ${ }^{82} \mathrm{Rb}$-PET for assessment of perfusion. (6) Adenosine stress imaging reflects the relative perfusion distribution within the major coronary vessel territories and usually not true ischemia, even though myocardium may become ischemic in cases of severe coronary stenosis due to proximal steel phenomenon. (7) The limited number of vessels $(n=17)$ where $i F R / F F R$ was performed and the absence of quantitative CMR data limits the ability to make general conclusions on the relation between invasive flow measurements, CMR and MFR by cardiac PET.

\section{NEW KNOWLEDGE GAINED}

The present paper emphasizes the need for quantitative non-invasive assessment of myocardial perfusion as well as for increased use of invasive flow reserve measurements during coronary angiography, in patients with suspected stable CAD.

\section{CONCLUSION}

The limited performance of qualitative assessment of the presence of stable CAD with CMR and CA, when related to quantitative ${ }^{13} \mathrm{~N}-\mathrm{NH}_{3}$ cardiac PET, shows the need for fully quantitative assessment of myocardial perfusion and the use of invasive flow reserve measurements for CA, to confirm the need of elective revascularization in patients with suspected CAD.

\section{Disclosures}

Sources of funding include Swedish Heart Lung Foundation, Institution of Clinical Sciences in Lund (IKVL) and Region of Scania. Haikan Arheden is stockholder in Imacor AB, Lund, Sweden. Marcus Carlsson and Henrik Engblom have recieved consultancy fees from Imacor AB, Lund, Sweden, for analysis of cardiac MRI. The remaining co-authors have no disclosures. No other potential conflicts of interest relevant to this article exist.

\section{Open Access}

This article is distributed under the terms of the Creative Commons Attribution 4.0 International License (http://creativ ecommons.org/licenses/by/4.0/), which permits unrestricted use, distribution, and reproduction in any medium, provided you give appropriate credit to the original author(s) and the source, provide a link to the Creative Commons license, and indicate if changes were made.

\section{References}

1. Task Force M, Montalescot G, Sechtem U, et al. 2013 ESC guidelines on the management of stable coronary artery disease: The Task Force on the management of stable coronary artery disease of the European Society of Cardiology. Eur Heart J 2013;34:2949-3003.

2. Edenbrandt L, Ohlsson M, Tragardh E. Prognosis of patients without perfusion defects with and without rest study in myocardial perfusion scintigraphy. EJNMMI Res 2013;3:58.

3. Metz LD, Beattie M, Hom R, Redberg RF, Grady D, Fleischmann KE. The prognostic value of normal exercise myocardial perfusion imaging and exercise echocardiography: A meta-analysis. J Am Coll Cardiol 2007;49:227-37.

4. Authors/Task Force members, Windecker S, Kolh P, et al. ESC/ EACTS Guidelines on myocardial revascularization: The Task Force on Myocardial Revascularization of the European Society of Cardiology (ESC) and the European Association for Cardio-Thoracic Surgery (EACTS)Developed with the special contribution of the European Association of Percutaneous Cardiovascular Interventions (EAPCI). Eur Heart J. 2014;2014:2541-619.

5. Fihn SD, Blankenship JC, Alexander KP, et al. 2014 ACC/AHA/ AATS/PCNA/SCAI/STS focused update of the guideline for the diagnosis and management of patients with stable ischemic heart disease: A report of the American College of Cardiology/American Heart Association Task Force on Practice Guidelines, and the American Association for Thoracic Surgery, Preventive Cardiovascular Nurses Association, Society for Cardiovascular Angiography and Interventions, and Society of Thoracic Surgeons. J Am Coll Cardiol 2014;64:1929-49.

6. Danad I, Szymonifka J, Twisk JWR, et al. Diagnostic performance of cardiac imaging methods to diagnose ischaemia-causing coronary artery disease when directly compared with fractional flow reserve as a reference standard: A meta-analysis. Eur Heart J 2017;38:991-8.

7. National Institute of Health and Care Excellence. Stable angina: management. https://www.nice.org.uk/guidance/cg126\#.

8. Carlsson M. The impacts on healthcare when coronary angiography as the reference method for diagnostic accuracy of coronary 
artery disease is replaced by fractional flow reserve! Eur Heart J 2017;38:999-1001.

9. Hannan EL, Samadashvili Z, Cozzens K, et al. Appropriateness of diagnostic catheterization for suspected coronary artery disease in New York State. Circ Cardiovasc interv 2014;7:19-27.

10. Greenwood JP, Maredia N, Younger JF, et al. Cardiovascular magnetic resonance and single-photon emission computed tomography for diagnosis of coronary heart disease (CE-MARC): A prospective trial. Lancet 2012;379:453-60.

11. Greenwood JP, Ripley DP, Berry C, et al. Effect of care guided by cardiovascular magnetic resonance, myocardial perfusion scintigraphy, or NICE guidelines on subsequent unnecessary angiography rates: The CE-MARC 2 Randomized Clinical Trial. JAMA 2016;316:1051-60.

12. Schwitter J, Wacker CM, van Rossum AC, et al. MR-IMPACT: Comparison of perfusion-cardiac magnetic resonance with singlephoton emission computed tomography for the detection of coronary artery disease in a multicentre, multivendor, randomized trial. Eur Heart J 2008;29:480-9.

13. Schwitter J, Wacker CM, Wilke N, et al. MR-IMPACT II: Magnetic Resonance Imaging for Myocardial Perfusion Assessment in Coronary artery disease Trial: Perfusion-cardiac magnetic resonance vs. single-photon emission computed tomography for the detection of coronary artery disease: A comparative multicentre, multivendor trial. Eur Heart J 2013;34:775-81.

14. Kellman P, Hansen MS, Nielles-Vallespin S, et al. Myocardial perfusion cardiovascular magnetic resonance: Optimized dual sequence and reconstruction for quantification. J Cardiovasc Magn Reson 2017;19:43.

15. Mordini FE, Haddad T, Hsu LY, et al. Diagnostic accuracy of stress perfusion CMR in comparison with quantitative coronary angiography: Fully quantitative, semiquantitative, and qualitative assessment. JACC Cardiovasc Imaging 2014;7:14-22.

16. Engblom H, Xue H, Akil S, et al. Fully quantitative cardiovascular magnetic resonance myocardial perfusion ready for clinical use: A comparison between cardiovascular magnetic resonance imaging and positron emission tomography. J Cardiovasc Magn Reson 2017;19:78

17. DeGrado TR, Hanson MW, Turkington TG, et al. Estimation of myocardial blood flow for longitudinal studies with $13 \mathrm{~N}$-labeled ammonia and positron emission tomography. J Nucl Cardiol 1996;3:494-507.

18. Herzog BA, Husmann L, Valenta I, et al. Long-term prognostic value of $13 \mathrm{~N}$-ammonia myocardial perfusion positron emission tomography added value of coronary flow reserve. J Am Coll Cardiol 2009;54:150-6.
19. Fiechter M, Ghadri JR, Gebhard C, et al. Diagnostic value of $13 \mathrm{~N}$-ammonia myocardial perfusion PET: added value of myocardial flow reserve. J Nucl Med 2012;53:1230-4.

20. Heiberg E, Sjogren J, Ugander M, Carlsson M, Engblom H, Arheden H. Design and validation of Segment-freely available software for cardiovascular image analysis. BMC Med Imaging 2010;10:1.

21. Engblom H, Tufvesson J, Jablonowski R, et al. A new automatic algorithm for quantification of myocardial infarction imaged by late gadolinium enhancement cardiovascular magnetic resonance: Experimental validation and comparison to expert delineations in multi-center, multi-vendor patient data. J Cardiovasc Magn Reson 2016;18:27.

22. Tonino PA, De Bruyne B, Pijls NH, et al. Fractional flow reserve versus angiography for guiding percutaneous coronary intervention. N Engl J Med 2009;360:213-24.

23. Shiode N, Okimoto T, Tamekiyo H, et al. A Comparison between the instantaneous wave-free ratio and resting distal coronary artery pressure/aortic pressure and the fractional flow reserve: The diagnostic accuracy can be improved by the use of both indices. Intern Med 2017;56:749-53.

24. Jaarsma C, Leiner T, Bekkers SC, et al. Diagnostic performance of noninvasive myocardial perfusion imaging using single-photon emission computed tomography, cardiac magnetic resonance, and positron emission tomography imaging for the detection of obstructive coronary artery disease: a meta-analysis. J Am Coll Cardiol 2012;59:1719-28.

25. Salerno M, Beller GA. Noninvasive assessment of myocardial perfusion. Circ Cardiovasc Imaging 2009;2:412-24.

26. White $\mathrm{CW}$, Wright CB, Doty DB, et al. Does visual interpretation of the coronary arteriogram predict the physiologic importance of a coronary stenosis? N Engl J Med 1984;310:819-24.

27. Camici PG, Crea F. Coronary microvascular dysfunction. N Engl J Med 2007;356:830-40.

28. Stuijfzand WJ, Uusitalo V, Kero T, et al. Relative flow reserve derived from quantitative perfusion imaging may not outperform stress myocardial blood flow for identification of hemodynamically significant coronary artery disease. Circ Cardiovasc Imaging 2015;8:e02400.

29. Gupta A, Taqueti VR, van de Hoef TP, et al. Integrated noninvasive physiological assessment of coronary circulatory function and impact on cardiovascular mortality in patients with stable coronary artery disease. Circulation 2017;136:2325.

30. Kaufmann PA, Camici PG. Myocardial blood flow measurement by PET: Technical aspects and clinical applications. J Nucl Med 2005;46:75-88. 